\title{
Primary Osteosarcoma Arising From Skull- A Case Report
}

\author{
Authors \\ Dr K Srinivas Rao ${ }^{1}$, Dr Lalitha Reddy.K ${ }^{1}$, Dr Syed Fathima ${ }^{1}$, Dr Sanjay.R, \\ Dr Milaap Shah ${ }^{2}$ \\ ${ }^{1 *}$ Department of Radiation Oncology, Yashoda Hospital Secundrabad, Telangana, India \\ ${ }^{2}$ Department of Pathology, Yashoda Hospital Secundrabad, Telangana, India \\ Corresponding Author \\ Dr Syed Fathima
}

Address: 10-3-36 Ward no:130 First floor Mahendra Hills, Addagutta, East Maredpally, Secundrabad,

500026, Telangana, India

Abstract
Primary osteosarcomas of skull which occur de novo are less frequent. Only 150 cases of osteosarcoma
skull have been reported. ${ }^{[7]}$ A 20 year old female presented with osteosarcoma in the Right temporal
bone as painless temporal swelling, which rapidly enlarged since 1 month. She underwent Craniotomy
and Biopsy elsewhere, Final Histopathology report was suggestive of Primary osteosarcoma.

\section{Introduction}

Osteosarcoma is most common bone tumor which typically occurs in extremities. ${ }^{[2]}$ Only $6 \%$ to $8 \%$ of osteosarcomas arise from skull and hence a rare entity. ${ }^{[2]}$ Osteosarcomas of skull presents late compared to osteosarcomas of long bones, usually in third to fourth decade with no gender predilection. $^{[2]}$ Because of rare condition, we report a case of osteosarcoma arising de novo from the temporal bone in a 20 year old female who presented with swelling in the Right temporal region (Fig:1) and headache. We describe clinical symptoms and the imaging features of this rare tumor and provide a brief review of the literature.

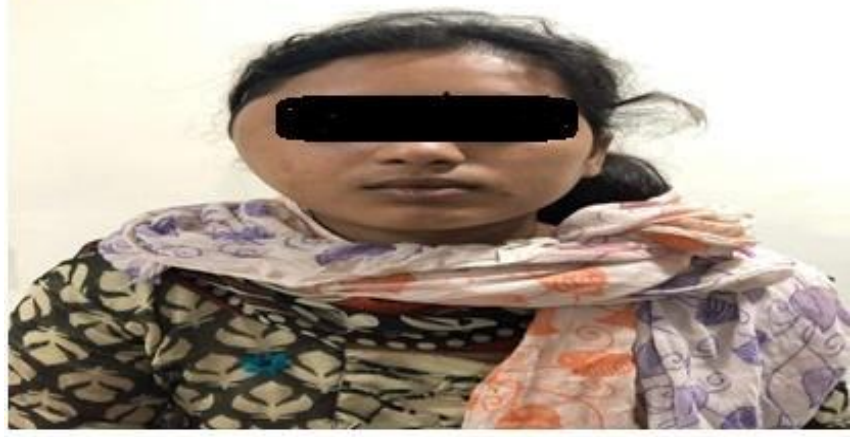

Fig 1: Patient has Temporal swelling.

\section{Case Report}

A 20 year old female was diagnosed with Right temporal extraaxial lesion underwent Right temporal craniotomy and biopsy of the lesion elsewhere .Post op Histopathology was suggestive of ?osteoblastoma / ?osteosarcoma 
Patient now presented to our institute with Right temporal fossa swelling which was pain full. Pain was moderate and continuous type. On physical examination patient had firm to hard diffuse swelling in Right temporal fossa. Tenderness was present. A healthy temporal craniotomy scar present. No neurological deficit on CNS examination. (Fig:1) Patient has no comorbidities, and no history of any treatment (H/oirradiation, H/o chemotherapy), trauma and pagets disease.

Evaluated further with MRI Brain

T2 weighted MR imaging revealed ill defined mass lesion in Right anterior aspect of temporal region which is seen terminating along Right temporal fossa at craniotomy site. Post gadolinium scan showed diffuse enhancement with central non enhancing area

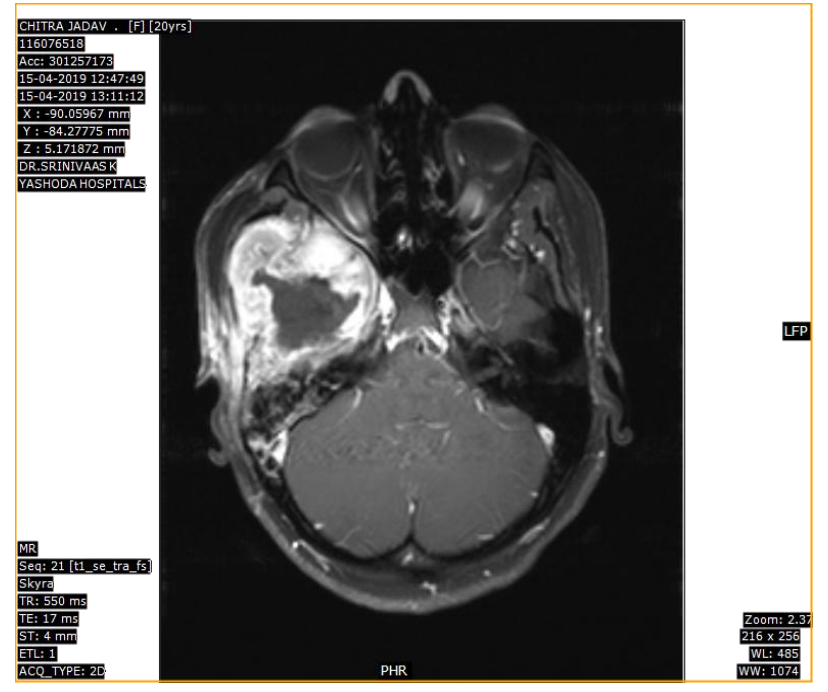

Fig 2: MRI Brain Image showing post gadolinium enhancement surrounded by hemosiderin deposition probably resection cavity, lesion approximately measures $6.5 \times 5.5 \mathrm{~cm}$ which is seen extending along right temporal soft tissue and scalp region. (Fig:2) MR Spectroscopy shows reduced NAA and choline peaks with relatively preserved NAA peaks within enhancing components of the lesion.

CT scan of Temporal bone: Showed large lytic lesion measuring $6.8 \times 5.8 \mathrm{~cm}$ with large soft tissue component and calcification/ossification involving right greater wing of sphenoid temporal bone with irregular heterogenous enhancement the lesion showed intracranial extension and inferiorly involvement of Temperomandibular joint.

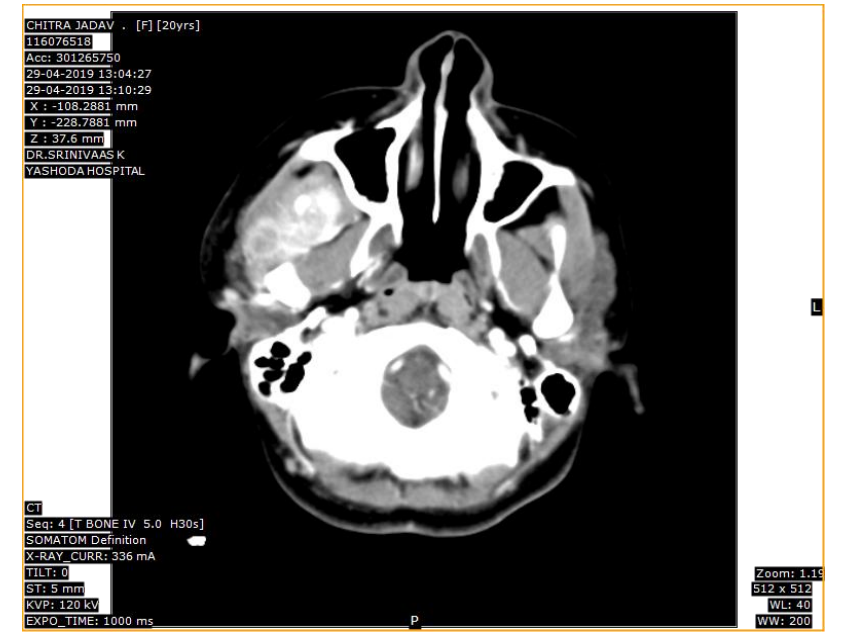

Fig 3: CT Temporal bone Showing Heterogenous enhancement of right temporal bone
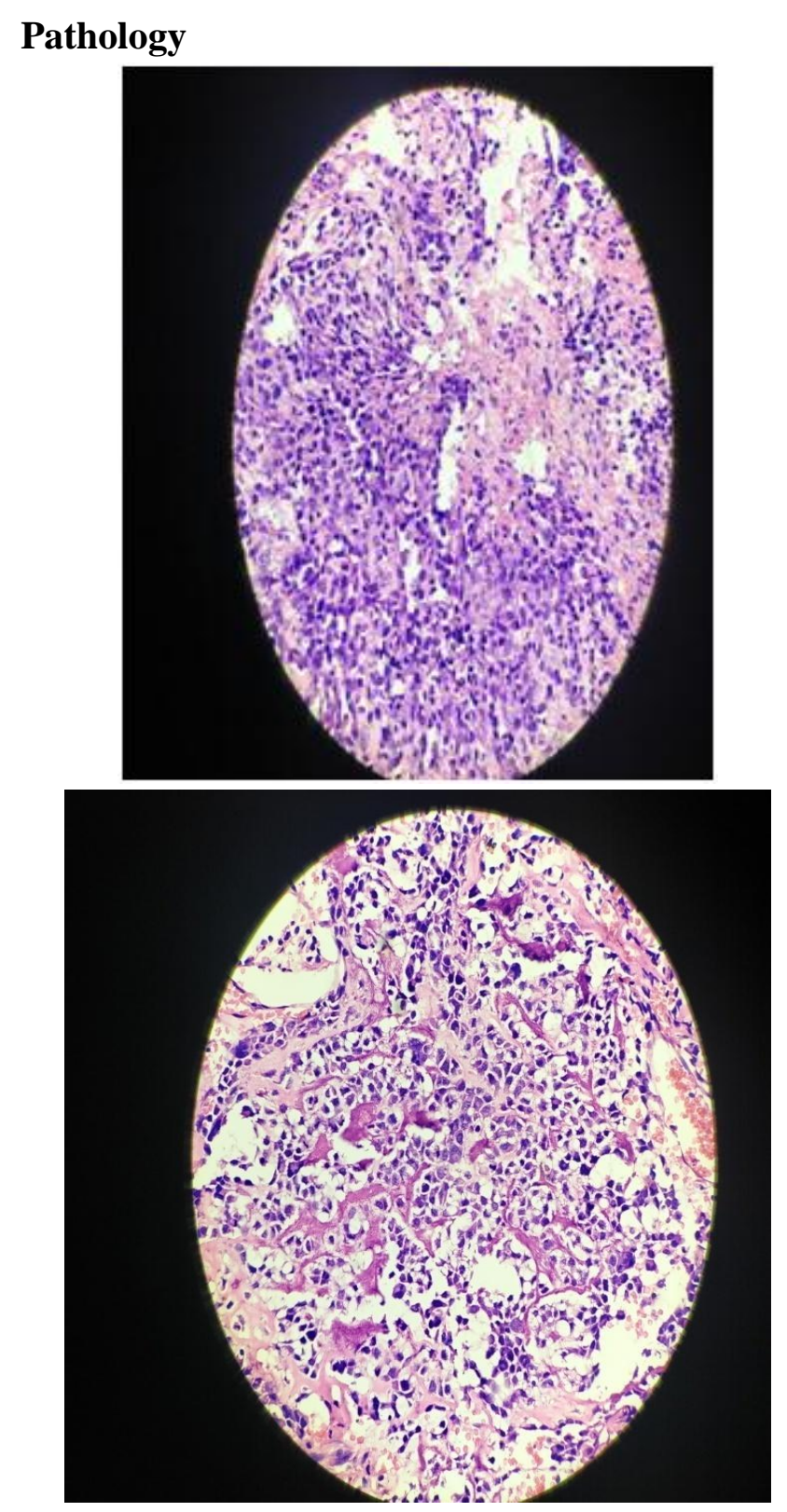

Fig 3: HPE showing filigree pattern of osteoid matrix without osteoblastic rimming. 


\section{Description}

Pleomorphic cells with marked atypia with filigree pattern of osteiod matrix. Osteiod is devoid of osteoblastic rimming.

\section{Discussion}

Osteosarcoma is a common tumor in extremities but in skull region it is very rare. Incidence of primary osteosarcoma is rare (1\% to $2 \%$ of all skull tumors). The occurrence of osteosarcoma in the skull peaks in the third decade. ${ }^{[2]}$ Common risks factors for osteosarcoma of skull is similar to that of osteosarcoma of the long bones, such as radiation exposure, Paget disease, Li-Fraumeni syndrome, and Other bone abnormalities, such as fibrous dysplasia, multiple osteochondromatosis, chronic osteomyelitis, myositis ossificans, and trauma are also associated conditions. ${ }^{[2]}$ Sedondary osteosarcoma occurs most commonly with pagets disease, has higher recurrence rate and are usually more aggressive. ${ }^{(7)}$

Clinical presentation of skull osteosarcoma is based on site of tumor involved, it usually presents as slow growing painless swelling, Other symptoms as cranial nerve palsies, headache and visual impairments can be seen. ${ }^{(2)}$ Histologically osteosarcoma has spindle shaped cells with immature bone formation. Histopathological type consists of Osteoblastic which is most common among all subtypes others include chondroblastic, fibroblastic, telangiectatic, parosteal, periosteal, and small cell osteosarcomas. ${ }^{(7)}$ If osteosarcoma of bone suspected, infiltrative lesion identified on plain radiograph should be followed by an MRI to further characterize the tumor location and extension, Imaging with MRI and CT may reveal bone growth with lytic regions and periosteal remodeling, as seen in osteosarcomas of other areas. ${ }^{(7)}$ Core needle biopsy or open biopsy can be performed $^{(1)}$ to confirm the diagnosis.

As osteosarcoma of skull is a rare disease, correct diagnosis and proper treatment plans are difficult. Upon diagnosis of osteosarcoma patients should undergo maximum safe resection of detectable disease and followed by adjuvant therapy (chemotherapy/radiotherapy). ${ }^{(7)}$ Surgical resection is beneficial in patients with tumors that are confined to the periosteum and locally invasive disease. Age at time of diagnosis, surgical resection are clinically significant survival determinants for osteosarcoma. Post surgical Local recurrence has poor prognosis, Increased Tumor size responds less to chemotherapy and a poor prognosis indicator. Currently, the combination of methotrexate, Adriamycin, and cisplatin has become standard of care. Radiotherapy may also be administered, but osteosarcoma is relatively radioresistant, this may more commonly be reserved for inoperable or more advanced cases. ${ }^{(3)}$ Metastasis seen most commonly to Lung associated with worse Prognosis. ${ }^{(4)}$

\section{References}

1. Martin E, Senders JT, terWengel PV, Smith TR, Broekman ML. Treatment and survival of osteosarcoma and Ewing sarcoma of the skull: a SEER database analysis. Actaneurochirurgica. 2019 Feb 13;161(2):317-25.

2. Wu G, Liang Q, Liu Y. Primary osteosarcoma of frontal bone: A case report and review of literature. Medicine. 2017 Dec;96(51).

3. Ta HT, Dass CR, Choong PF, Dunstan DE. Osteosarcoma treatment: state of the art. Cancer and Metastasis Reviews. 2009 Jun 1;28(1-2):247-63.

4. Arshi A, Sharim J, Park DY, Park HY, Yazdanshenas H, Bernthal NM, Shamie AN. Prognostic determinants and treatment outcomes analysis of osteosarcoma and Ewing sarcoma of the spine. The Spine Journal. 2017 May 1;17(5):645-55.

5. Gonzalez ME, Raghavan $\mathrm{P}$, Cho B, Muttikkal TJ, Rehm PK. Primary osteogenic osteosarcoma of the ethmoid sinus in an adolescent: case report. Journal 
of radiology case reports. 2016 Feb;10 (2):1.

6. Chung LH, Wu PK, Chen CF, Weng HK, Chen TH, Chen WM. Pathological fractures in predicting clinical outcomes for patients with osteosarcoma. BMC musculoskeletal disorders. 2016 Dec;17(1):503.

7. Hadley C, Gressot LV, Patel AJ, Wang LL, Flores RJ, Whitehead WE, Luerssen TG, Jea A, Bollo RJ. Osteosarcoma of the cranial vault and skull base in pediatric patients: Report of 3 cases. Journal of Neurosurgery: Pediatrics. 2014 Apr 1;13(4):380-7. 